\title{
Importancia del monitoreo de procesos biogeoquímicos en lagunas costeras: Área Natural Protegida estero El Soldado como estudio de caso
}

\author{
The importance of monitoring biogeochemical processes in coastal lagoons: \\ Estero El Soldado Protected Natural Area as a case study
}

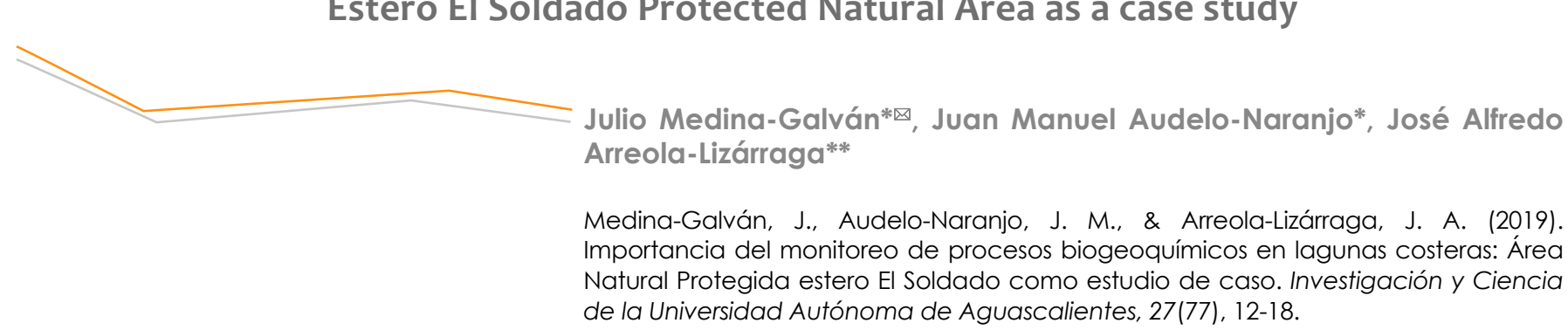

RESUMEN

Esta contribución presenta estimaciones de los flujos de nutrientes y el metabolismo neto en una laguna costera prístina del Golfo de California. Durante primavera se realizaron muestreos de variables del agua en la laguna y el mar adyacente. Los flujos de nutrientes y el metabolismo neto del ecosistema fueron estimados con el modelo biogeoquímico propuesto por el programa LOICZ (Interacciones Tierra Océano en la Zona Costera). La tasa de renovación del agua fue de 3 días. La laguna El Soldado funcionó como fuente de nitrógeno (+61 mol día $\left.{ }^{-1}\right)$ y como sumidero de fósforo $(-42 \mathrm{~mol}$ día $\left.^{-1}\right)$. El metabolismo neto fue autótrofo $(73 \mathrm{mmol}$ $\mathrm{C} \mathrm{m}^{2} \mathrm{mes}^{-1}$ ) y dominó la fijación de nitrógeno. Estas observaciones en la laguna El Soldado ayudan al entendimiento de los procesos biogeoquímicos y

Palabras clave: procesos biogeoquímicos; flujos de nutrientes; metabolismo neto del ecosistema; lagunas costeras.

Keywords: biogeochemical processes; nutrients fluxes; net ecosystem metabolism; coastal lagoons.

Recibido: 3 de julio de 2018, aceptado: 4 de marzo de 2019

* Facultad de Ciencias del Mar, Universidad Autónoma de Sinaloa. Paseo Claussen S/N, Apdo. Postal 610, C. P. 82000, Mazatlán, Sinaloa, México. Correo electrónico: jmedinag.facimar@uas.edu.mx; Jmaudelo@gmail. com. ORCID: http://orcid.org/0000-0002-1168-9339; http://orcid. org/0000-0003-2028-8710

** Centro de Investigaciones Biológicas del Noroeste. Km. 2.35 camino al Tular, Estero Bacochibampo, C. P. 85454, Guaymas, Sonora, México. Correo electrónico: aarreola04@cibnor.mx. ORCID: http://orcid. org/0000-0002-0936-8886

$凶$ Autor para correspondencia exhibe la importancia de su monitoreo a través del ciclo anual, así como en periodos de largo plazo.

\section{ABSTRACT}

This contribution presents estimates of nutrient fluxes and net metabolism in a pristine coastal lagoon of the Gulf of California. During spring, water variables were sampled in the lagoon and the adjacent sea. The biogeochemical model proposed by the LOICZ program (Land-Ocean Interactions in the Coastal Zone) estimated the nutrient fluxes and the net metabolism of the ecosystem. The water renewal rate was 3 days. The El Soldado lagoon functioned as a source of nitrogen (+61 mol day $\left.{ }^{-1}\right)$ and as a phosphorus basin $\left(-42 \mathrm{~mol} \mathrm{day}^{-1}\right)$. The net

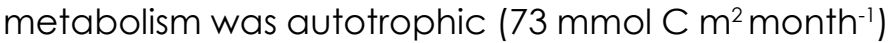
and dominated nitrogen fixation. These observations in the El Soldado lagoon, help to understand the biogeochemical processes and show the importance of their monitoring throughout the annual cycle, as well as in long term periods.

INTRODUCCIÓN

La biogeoquímica se define como un campo integrador en el cual se estudian los ciclos que incluyen las interacciones entre procesos biológicos, químicos y geológicos (Bianchi, 2007). De esta forma, el estudio de los procesos biogeoquímicos permite conocer las conexiones entre la biósfera, la atmósfera y la hidrósfera, así como las interacciones entre los elementos que las componen (Esteve \& Jaén, 2013). 
En la zona costera la interacción de procesos biológicos, químicos y geológicos determinan los flujos de sedimentos, nutrientes, materia orgánica y contaminantes entre ecosistemas. En particular, las lagunas costeras y estuarios, mediante procesos biogeoquímicos, proporcionan valiosos servicios ecosistémicos como reciclaje de nutrientes, descomposición de materia orgánica y remoción de contaminantes (Barbier et al., 2011; Piehler \& Smith, 2011). Estos cuerpos de agua presentan diferentes capacidades para transformar los nutrientes introducidos, debido a sus diferencias en ubicación geográfica (tropical, templada, y otros), superficie, profundidad y tasa de intercambio de agua con el mar adyacente (Dettmann, 2001; Smith et al., 2003).

Los flujos implican tasas de transferencia y se definen como la cantidad de material que se transporta de un cuerpo de agua a otro durante un periodo de tiempo determinado (masa/tiempo; masa/ área/tiempo). Un sistema fuente exporta materiales y un sistema sumidero los importa (Bianchi, 2007).

Por otro lado, el metabolismo neto del ecosistema (MNE) se define como la diferencia entre la producción primaria y la respiración de un sistema acuático (Odum, 1969). El MNE es positivo (autótrofo) cuando la producción primaria excede a la respiración y es negativo (heterótrofo) cuando la respiración excede a la producción primaria (Kemp \& Testa, 2011).

Un asunto relevante es que mediante modelos biogeoquímicos es posible evaluar las capacidades que tienen los estuarios y lagunas costeras para transformar, acumular y exportar materia disuelta (sal, nutrientes) y también permiten conocer el metabolismo neto del ecosistema (Gordon et al., 1996; Swaney, Smith, \& Wulff, 2011), de tal forma que el entendimiento de los flujos de nutrientes y el metabolismo neto a través de modelos biogeoquímicos sencillos puede proveer herramientas para el mejor manejo de la zona costera (Cervantes-Duarte, 2016).

El objetivo del presente estudio fue presentar la estimación de procesos biogeoquímicos mediante un modelo de balance de masas, utilizando como estudio de caso el comportamiento de los flujos de nutrientes y metabolismo neto durante la primavera en el estero El Soldado, área natural protegida del Golfo de California.

\section{MATERIALES Y MÉTODOS}

\section{Área de estudio}

El estero El Soldado es una laguna costera localizada en la costa este del Golfo de California (figura 1). Por su origen geológico se clasifica como tipo III-B: erosión diferencial cañón rocoso inundado (Lankford, 1977). El área de espejo de agua es de $1.6 \mathrm{~km}^{2}$, tiene profundidad promedio de $0.60 \mathrm{~m}$ y se comunica con el mar a través de una boca de 40 $\mathrm{m}$ de ancho y $3 \mathrm{~m}$ de profundidad. Las mareas son mixtas con predominancia de semidiurnas (Russell, 1979). La amplitud de marea es de 1 m (Filloux, 1973) y clasifica como laguna micromareal (Davies et al., 1964). El tipo de clima es muy árido y seco. La temperatura promedio anual es de $25^{\circ} \mathrm{C}$. La tasa de precipitación anual es $<300 \mathrm{~mm}$ y la mayor parte ocurre en verano (García, 2004). Las escorrentías de lluvias son el único aporte de agua dulce a la laguna.

El estero El Soldado es un área natural protegida del estado de Sonora con la categoría de zona sujeta a conservación ecológica (Gobierno del Estado de Sonora, 18 de mayo de 2006).

\section{Trabajo de campo y análisis de muestras}

Los muestreos se realizaron en primavera (abril de 2009) con dos campañas, una durante mareas vivas y otra durante mareas muertas. Se establecieron

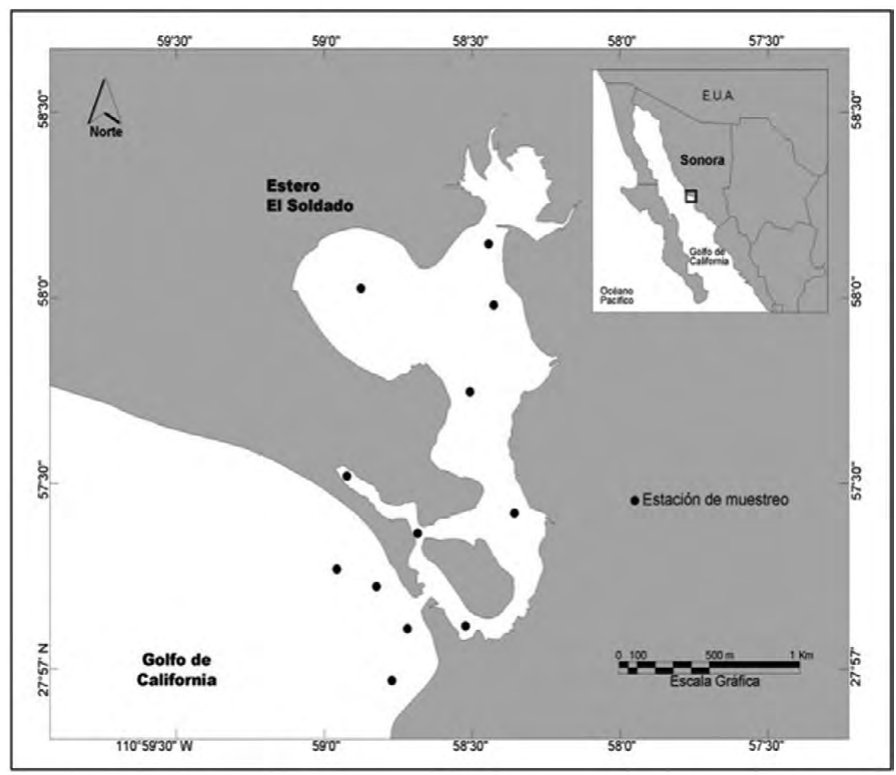

Figura 1. Área de estudio y sitios de muestreo. Elaboración propia. 
issn 1665-4412, e-issn 2521-9758

Medina-Galván, J., Audelo-Naranjo, J. M., \& Arreola-Lizárraga, J. A. ocho sitios de muestreo en la laguna y cuatro en el mar adyacente. En cada sitio se registraron in situ la temperatura, la salinidad y el oxígeno disuelto del agua con un equipo autónomo multisensor marca Hydrolab modelo Datasonde ${ }^{\circledR}$ y se recolectó una muestra de 1 I de agua subsuperficial en un recipiente de plástico con cierre hermético para determinar la concentración de nutrientes (nitrito, nitrato, amonio y ortofosfatos). Las muestras de agua fueron mantenidas en hielera $\mathrm{a} \sim 4^{\circ} \mathrm{C}$ durante su transporte al laboratorio. Para la determinación de los mencionados nutrientes se filtraron las muestras de agua y se siguieron las técnicas de Strickland y Parsons (1972).

\section{Descripción del modelo biogeoquímico}

Los flujos de nutrientes y el metabolismo neto del ecosistema se estimaron mediante el modelo biogeoquímico de Interacciones Tierra Océano en la Zona Costera (LOICZ, por sus siglas en inglés), descrito por Gordon et al. (1996). El modelo se construye en tres etapas: (1) balance de agua, (2) balance de sal, y (3) balance de nutrientes (figura 2). El volumen de mezcla es resultado de los balances de agua y sal, de tal forma que el tiempo de residencia del

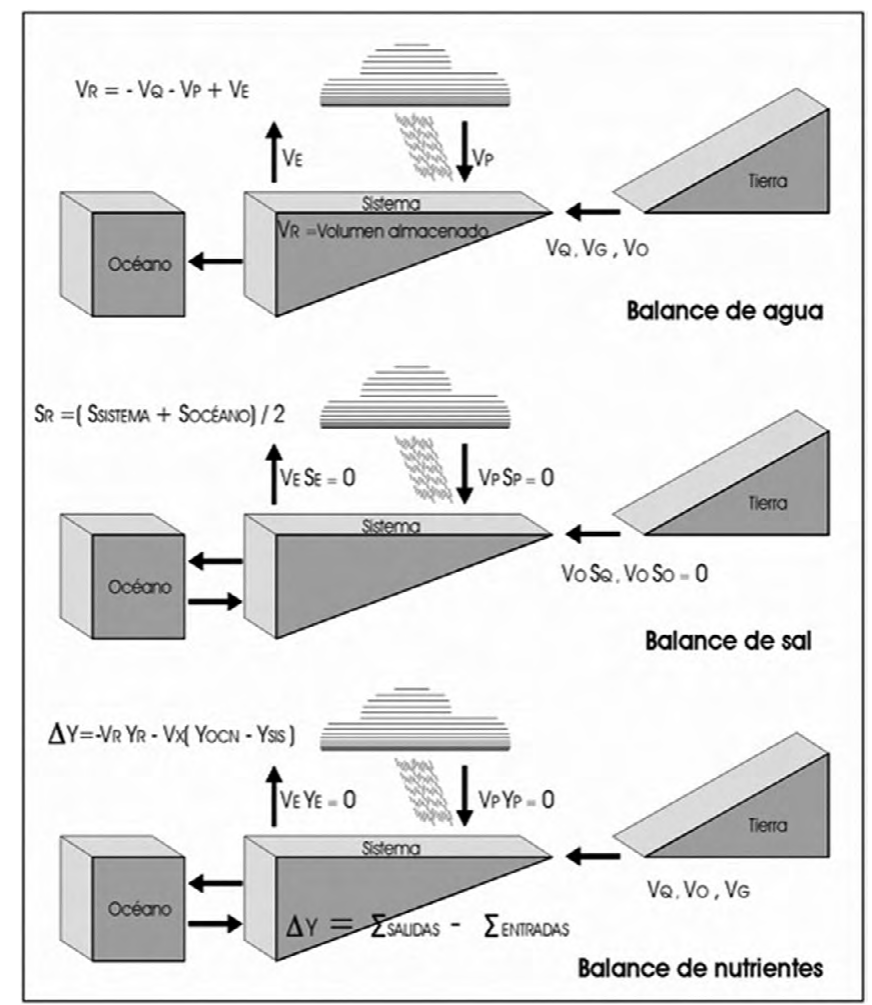

Figura 2. Procedimiento para estimar los balances de agua, sal y nutrientes.

Elaboración propia. agua es obtenido de dividir el volumen del sistema entre el volumen de mezcla.

De acuerdo a la figura 2:

$V Q \quad$ son aguas residuales agrícolas (en este caso se asumió cero).

VP son lluvias.

VG es el aporte del manto freático (en este caso se asumió cero).

vo son otros aportes (en este caso se asumió cero).

VE es evaporación.

VR es el volumen residual, que representa el volumen de agua a compensar.

SR es la salinidad asociada al flujo residual y representa el promedio entre la salinidad de la laguna El Soldado (SSIS) y la salinidad del mar adyacente (SOCN).

$\checkmark X \quad$ es el volumen de mezcla, que compensa el agua y sal en el balance.

$\Delta Y$ es el flujo del material no conservativo (Fósforo Inorgánico Disuelto FID y Nitrógeno Inorgánico Disuelto NID).

YR es el valor promedio del material no conservativo entre dos fronteras.

YOCN es el valor promedio del material no conservativo del océano.

YSIS es el valor promedio del material no conservativo del sistema.

\section{Estimación del metabolismo neto, fijación de nitrógeno y desnitrificación}

El metabolismo neto del ecosistema (MNE), que representa la diferencia entre la producción primaria (p) y la respiración (r) fue estimado con la siguiente ecuación:

$(p-r)=-\Delta F I D \times(C: P) p a r t$

donde:

(C:P)part es la razón carbono a fósforo en la materia orgánica particulada que está siendo reciclada en el sistema. En la estimación se asume el índice molar de Redfield (1934), que corresponde a C:P 106:1.

RESULTADOS

Las variables ambientales del agua, temperatura y salinidad presentaron valores promedios más altos en la laguna con respecto al mar adyacente; las concentraciones de oxígeno disuelto fueron más altas en el mar adyacente; las concentraciones de 
Tabla 1

Variables del agua en el estero El Soldado y mar adyacente durante primavera

\begin{tabular}{|c|c|c|c|c|}
\hline \multirow[t]{2}{*}{ Variable } & \multicolumn{2}{|c|}{ Estero El Soldado $\quad(n=16)$} & \multicolumn{2}{|c|}{ Mar adyacente $\quad(n=8)$} \\
\hline & Mín.- Máx. & Prom. \pm D. E. & Mín.- Máx. & Prom. \pm D. E. \\
\hline Temperatura ${ }^{\circ} \mathrm{C}$ & $19.84-25.51$ & $22.40-1.98$ & $18.84-20.34$ & $19.6-0.67$ \\
\hline Salinidad ups & $36.97-39.11$ & $38.10-0.72$ & $36.91-37.19$ & $37-0.11$ \\
\hline Oxígeno disuelto mg & $6.67-7.81$ & $7.10-0.39$ & $7.30-8.94$ & $8.20-0.61$ \\
\hline $\mathrm{N}-\mathrm{NO}_{2} \mu \mathrm{M}$ & $0.02-0.13$ & $0.09-0.04$ & $0.04-0.12$ & $0.08-0.03$ \\
\hline $\mathrm{N}-\mathrm{NO}_{3} \mu \mathrm{M}$ & $0.07-0.31$ & $0.13-0.09$ & $0.07-0.30$ & $0.12-0.10$ \\
\hline $\mathrm{N}-\mathrm{NH}_{4} \mu \mathrm{M}$ & $0.18-2.63$ & $0.86-0.55$ & $0.20-1.49$ & $0.68-0.44$ \\
\hline$N I D \mu M$ & $0.40-2.88$ & $1.09-0.56$ & $0.32-1.49$ & $0.88-0.45$ \\
\hline FID $\mu \mathrm{M}$ & $1.10-2.02$ & $1.30-0.44$ & $1.10-1.85$ & $1.40-0.28$ \\
\hline
\end{tabular}

Nota: Elaboración propia.

nitrito, nitrato y amonio fueron mayores en la laguna; las de fósforo inorgánico disuelto fueron similares entre la laguna y el mar (tabla 1).

\section{Balance de agua, sal y estimación de la tasa de renovación del agua}

El tiempo de renovación de agua estimado fue de 3 días. Los aportes de agua por lluvia son menores que la pérdida por evaporación. En primavera el agua que se evapora es compensada por la que ingresa al sistema desde el mar adyacente, ya que en el periodo de estudio el aporte de agua dulce por lluvia fue mínimo. La laguna no tiene aportes de agua por ríos ni tampoco por fuentes antropogénicas (tabla 2).

Tabla 2

Características hidrológicas de la laguna El Soldado

\begin{tabular}{lc}
\hline \multicolumn{1}{c}{ Concepto } & $\begin{array}{c}\text { Laguna } \mathrm{EL} \\
\text { Soldado }\end{array}$ \\
\hline Superficie $\left(\mathrm{km}^{2}\right)$ & 1.85 \\
Profundidad $(\mathrm{m})$ & 0.6 \\
Volumen del sistema $\left(\mathrm{m}^{3}\right)$ & $1,110,000$ \\
Precipitación $\left(\mathrm{m}^{3}\right.$ día $\left.^{-1}\right)$ & 37 \\
Evaporación $\left(\mathrm{m}^{3}\right.$ día $\left.^{-1}\right)$ & $-9,515$ \\
Volumen de mezcla $\left(\mathrm{m}^{3}\right.$ día $\left.^{-1}\right)$ & 335,658 \\
Tiempo de residencia (días) & 3 \\
Otros aportes ( $\mathrm{m}^{3}$ día $\left.^{-1}\right)$ & 0 \\
Aporte del mar al sistema $\left(\mathrm{m}^{3}\right.$ día $\left.^{-1}\right)$ & 9,478 \\
\hline
\end{tabular}

Nota: Elaboración propia.

\section{Flujos de nutrientes}

Los flujos de NID (nitrógeno inorgánico disuelto) mostraron que la laguna El Soldado exportó hacia el mar adyacente funcionando como fuente. Los flujos de FID (fósforo inorgánico disuelto) mostraron ingreso desde el mar adyacente hacia la laguna y esta funcionó como sumidero (figura 3).

\section{Metabolismo neto del ecosistema}

El metabolismo neto del ecosistema fue autótrofo, lo cual indica que el estero El Soldado está fijando carbono a través de la productividad primaria a una razón de $73.8 \mathrm{mmol} \mathrm{C} \mathrm{m}^{2} \mathrm{mes}^{-1}$ y la fijación de nitrógeno excedió a la desnitrificación a una tasa de 12 $\mathrm{mmol} \mathrm{m} \mathrm{mes}^{-1}$, las que se indican en la tabla 3 .

Tabla 3

Metabolismo neto y fijación-desnitrificación del estero

\begin{tabular}{lcc}
\hline \multicolumn{1}{c}{ Procesos (Unidades) } & MNE & Fij-Desnit \\
\hline $\mathrm{mmol} \mathrm{C} \mathrm{m}^{2} \mathrm{mes}^{-1}$ & 73.8 & - \\
$\mathrm{mmol} \mathrm{m}{ }^{2} \mathrm{mes}^{-1}$ & - & 12 \\
Condición & Autótrofo & Fijación \\
\hline Nota: Elaboración propia. & &
\end{tabular}

DISCUSIÓN

La estimación de flujos de nutrientes y del metabolismo neto durante condiciones de primavera en la laguna El Soldado (figura 4) mostró que este ecosistema se comportó como fuente de nitrógeno, como sumidero de fósforo y exhibió un metabolismo autótrofo. En particular la temperatura 
issn 1665-4412, e-issn 2521-9758

Medina-Galván, J., Audelo-Naranjo, J. M., \& Arreola-Lizárraga, J. A.

16

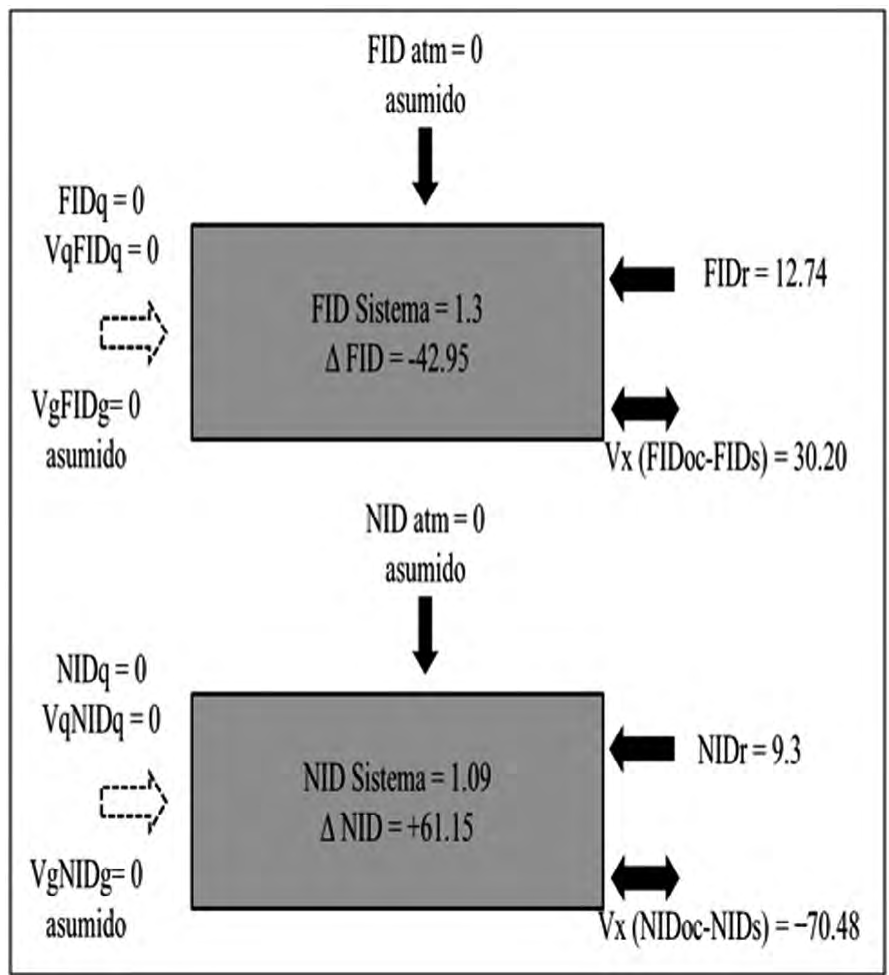

Figura 3. Flujos de nitrógeno y fósforo en el estero El Soldado. Flujos de nutrientes en mol día ${ }^{-1},(+)$ indica fuente, $(-)$ indica sumidero. Elaboración propia.

del agua observada en la laguna es resultado del intercambio de calor con la atmósfera, donde la variación anual de temperatura es extremosa por ser una región árida $>14^{\circ} \mathrm{C}$ (García, 2004), y debido a que la laguna es somera (profundidad promedio 0.6 $\mathrm{m}$ ) existe un rápido calentamiento o enfriamiento de la columna de agua determinados por los cambios diurnos y estacionales de irradiación solar.

La salinidad del agua en la laguna fue mayor a la observada en el mar adyacente debido al escaso aporte de agua dulce por precipitación $(<300$ $\mathrm{mm}$ año-1) y alta evaporación $(\sim 3,000 \mathrm{~mm}$ año-1 $)$. Los estuarios y lagunas costeras con intervalo de salinidad de 30-40 ups son clasificados como sistemas eurihalinos (Contreras-Espinoza \& Warner, 2004) y con base en los valores de salinidad observados, la laguna El Soldado corresponde a un sistema eurihalino. Los valores de oxígeno disuelto $>6 \mathrm{mg} \mathrm{l}^{-1}$ indicaron que es un sistema bien mezclado en primavera como resultado de la tasa de renovación de agua observada de $\sim 3$ días.

Las concentraciones de nitrógeno inorgánico disuelto en el agua fueron más altas en la laguna que en el mar adyacente y las de fósforo inorgánico disuelto resultaron similares entre ambos sistemas. Estas concentraciones observadas son características de primavera en lagunas costeras del Golfo de California (Arreola-Lizárraga et al., 2016).

Un factor importante del modelo biogeoquímico aplicado en este estudio es la estimación del tiempo de residencia o tasa de renovación del agua de la laguna (Gordon et al., 1996), la cual fue estimada en 3 días; este corto periodo es propiciado por las tasas de evaporación y el intercambio de agua con el mar adyacente por efecto de la marea, lo que implica que la laguna tiene buena capacidad de dilución y asimilación de nutrientes. Las tasas de renovación del agua de pocos días es característico en lagunas pequeñas cuyo volumen de agua se intercambia relativamente rápido con el mar adyacente mediante el flujo y reflujo de mareas (Smith et al., 2005).

En primavera, los flujos de nitrógeno mostraron que la laguna funcionó como fuente y exportó nutrientes al mar, el origen de este nitrógeno se atribuye al reciclaje interno por la remineralización de materia orgánica, tal como fue observado por Arreola-Lizárraga, Padilla-Arredondo, GaratuzaPayán y Ruiz-Ruiz (2015), Medina-Galván, PadillaArredondo y Arreola-Lizárraga (2018), así como por Valenzuela-Siu, Arreola-Lizárraga, Sánchez-Carrillo y Padilla-Arredondo (2007) en otras lagunas de esta región del Golfo de California. Los flujos de fósforo indicaron que la laguna funcionó como sumidero, explicado por las concentraciones altas de ortofosfato en el mar que sugieren ser un suministro importante de fósforo desde el mar a la laguna en primavera. Un proceso similar fue observado en la laguna Las Marites en época de secas (López-Monroy \& Troccoli-Ghinaglia, 2017).

El balance entre producción primaria y respiración en la laguna mostró que el metabolismo neto fue autótrofo en primavera, donde la producción primaria predominó ligeramente sobre la respiración a una tasa de $73.8 \mathrm{mmol} \mathrm{C} \mathrm{m}^{2}$ mes-1; este equilibrio entre autotrofía y heterotrofía se atribuyó a los procesos biogeoquímicos internos y al corto tiempo de renovación del agua que presentó la laguna. Esto coincide con lo observado por Kemp y Testa (2011) en el sentido de que las tasas rápidas de renovación del agua regulan el equilibrio entre fotosíntesis y respiración. 
Los procesos de fijación de nitrógeno y desnitrificación indicaron que dominó la fijación a una tasa de $12 \mathrm{mmol} \mathrm{m}{ }^{-2} \mathrm{mes}^{-1}$, estos resultados coinciden con lo observado en la laguna costera Algodones durante primavera (Arreola-Lizárraga et al., 2015), ubicada también en esta región del Golfo de California y la dominancia de la fijación de nitrógeno es un proceso que ha sido observado frecuentemente en lagunas costeras poco profundas (Smith \& Atkinson, 1994).

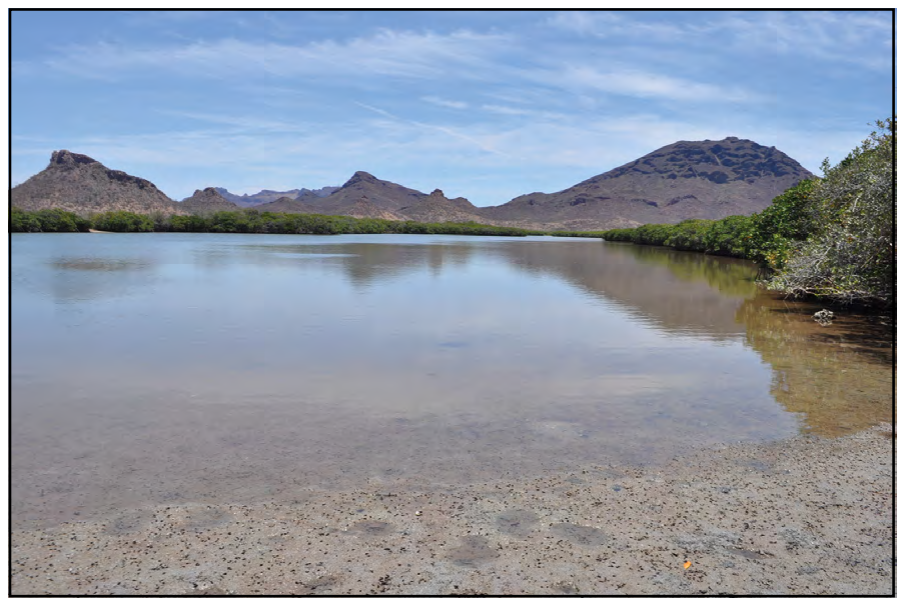

Figura 4. Área de estudio.

Fotografía del equipo de investigación.

\section{CONCLUSIONES}

En primavera las concentraciones de nitrógeno inorgánico disuelto en la laguna El Soldado fueron mayores con respecto a las observadas en el mar adyacente. Las concentraciones de fósforo inorgánico disuelto presentaron valores similares comparados con las del mar. La tasa de renovación del agua fue de 3 días. La laguna El Soldado funcionó como fuente de nitrógeno inorgánico disuelto y como sumidero de fósforo. El metabolismo neto fue autótrofo y dominó la fijación de nitrógeno. La estimación de los flujos de nutrientes y del metabolismo neto en la laguna El Soldado ayuda al entendimiento de los procesos biogeoquímicos y exhibe la importancia de su monitoreo a través del ciclo anual, así como en periodos de largo plazo. Un análisis comparativo del comportamiento de procesos biogeoquímicos entre lagunas costeras prístinas y receptoras de aguas residuales aportaría información útil para apoyar la gestión ambiental de estos valiosos ecosistemas costeros.

\section{Agradecimientos}

Los autores desean agradecer al personal técnico del CIBNOR, S. C. campus Guaymas María del Refugio López (análisis de muestras), Andrés Hernández (trabajo de campo), Gustavo Padilla (asesoría en el procesamiento del modelo LOICZ) y David Urías (elaboración de figuras); a los guardaparques del Área Natural Protegida estero El Soldado por su apoyo en el campo; al personal directivo de la Comisión de Desarrollo Sustentable del Estado de Sonora por todas las facilidades otorgadas para realizar el estudio. El presente estudio se realizó con financiamiento del proyecto 900-252 del CIBNOR. Julio Medina agradece al CONACYT por la beca 769282 otorgada para realizar estudios de doctorado.
- Arreola-Lizárraga, J. A., Padilla-Arredondo, G., GaratuzaPayán, J., \& Ruiz-Ruiz, T. M. (2015). Síntesis de procesos biogeoquímicos en la laguna costera La Luna, México (pp. 296-304). X Congreso de Ciencias del Mar MARCUBA.

- Arreola-Lizárraga, J. A., Padilla-Arredondo, G. Medina-Galván, J., Méndez-Rodríguez, L., Mendoza-Salgado, R., \& CordobaMatson, M. V. (2016). Analysis of hydrobiological responses to anthropogenic and natural influences in a lagoon system in the Gulf of California. Oceanological and Hydrobiological Studies, 45(1), 112-120.

- Barbier, E. B., Hacker, S. H., Kennedy, C., Koch, E. W., Stier, A. C., \& Silliman, B. R. (2011). The value of estuarine and coastal ecosystem services. Ecological Monographs, 81 (2), 169-193.

- Bianchi, T. S. (2007). Biogeochemistry of estuaries (720 pp.).
New York: Oxford University Press.

- Cervantes-Duarte, R. (2016). Nutrient fluxes and net metabolism in a coastal lagoon SW peninsula of Baja California, Mexico. Revista Bio Ciencias, 4 (2), 104-115.

- Contreras-Espinoza, F., \& Warner, B. G. (2004). Ecosystem characteristics and management considerations for coastal wetlands in Mexico. Hydrobiologia, 511, 233-245.

- Davies, J. L., Davies, J. L., Davies, J. H., Davies Gideon, J., Davies, L., \& Davies, M. (1964). A morphogenic approach to world shorelines. Zeitschrift für geomorphologie, 8, 27-42.

- Dettmann, E. H. (2001). Effect of water residence time on annual export and denitrification of nitrogen in estuaries: A model analysis. Estuaries, 24, 481-490. 
- Esteve, P., \& Jaén, M. (2013). El papel de los ciclos biogeoquímicos en el estudio de los problemas ambientales en Educación Secundaria. Investigación en la escuela, 80, 7788.

- Filloux, J. H. (1973). Tidal patterns and energy balance in the Gulf of California. Nature, 243, 217-221.

- García, E. (2004). Modificaciones al sistema de clasificación climática de Köppen (246 pp.). México, D. F.: Instituto de Geografía, Universidad Nacional Autónoma de México.

- Gobierno del Estado de Sonora. (18 de mayo de 2006). Declaratoria que establece área natural protegida bajo categoría zona sujeta a conservación ecológica donde se encuentra el estero El Soldado y áreas aledañas. Boletín Oficial, 177(40).

- Gordon, D. C. Jr., Boudreau, P. R., Mann, K. H., Ong, J. E., Silvert, W. L., Smith, S. V., ...Yanagi. T. (1996). LOICZ Biogeochemical Modelling Guidelines. LOICZ Reports \& Studies, 5, 1-96.

- Kemp, W.M., \& Testa, J.M. (201 1). Metabolic balance between ecosystem production and consumption. En E. Wolanskl, \& D. McLusky (Eds.), Treatise on estuarine and coastal science (pp. 83-118). New York: Academic Press.

- Lankford, R. R. (1977). Coastal lagoons of Mexico, their origin and classification. En M. Wiley (Ed.), Estuarine Processes (pp. 182-215). New York: Academic Press.

- López-Monroy, F., \& Troccoli-Ghinaglia, L. (2017). Modelaje de la interacción entre la laguna costera tropical las Marites (Isla de Margarita, Venezuela) y el mar Caribe adyacente. Saber, $29,534-545$.

- Medina-Galván, J., Padilla-Arredondo, G., \& ArreolaLizárraga, J. A. (2018). Flujos de nutrientes y metabolismo neto del estero Siuti (eurihalino, subtropical) en el Golfo de California. En: F. Paz, A. Velázquez, \& M. Rojo (Eds.), Serie Síntesis Nacionales: Estado Actual del Conocimiento del Ciclo del Carbono y sus Interacciones en México: Síntesis a 2018 (pp. 302-308, 678 pp.). Estado de México, México: Programa Mexicano del Carbono-Instituto Tecnológico de Sonora.

- Odum, E. P. (1969). The strategy of ecosystem development. Science, 164, 262-270.
- Piehler, M. F., \& Smyth, A. R. (2011). Habitat-specific distinctions in estuarine denitrification affect both ecosystem function and services. Ecosphere, 2(1), 1-17.

- Redfield, A. C. (1934). On the proportions of organic derivates in sea water and thir relation to the composition of plankton. En James Jhonstone Memorial Volumen (pp. 176-192). Liverpool, England: University Press of Liverpool.

- Russell, G. A. (1979). A seventeen month study of the meteorology, geology, hidrology and water chemistry of Laguna (estero) El Soldado, Guaymas, Sonora, México (146 pp.) Tech. Rep. Manitoba, Canadá: University of Manitoba.

- Smith, S. V., \& Atkinson, M. J. (1994). Mass balance of nutrient fluxes in coastal lagoons. En B. J. Kjerfve (Ed.), Coastal lagoon processes (pp. 133-155). New York: Elsevier.

- Smith, S. V., Buddemeier, R. W., Wulff, F., Swaney, D. P., Camacho-lbar, V. F.,David. L. T.,... Sandhei, P. (2005). C, N, $P$ fluxes in the coastal zone. En C. J. Crossland, H. H. Kremer, H. J. Lindeboom, J. I. Marshall-Crossland, \& M. D. A. Le Tissier (Eds.), Coastal Fluxes in the Anthropocene (pp. 95-143). Berlin, Germany: Springer-Verlag.

- Smith, S. V., Swaney, D. P., Talaue-McManus, L., Bartley, J. D., Sandhei, P. T., McLaughlin, C. J.,...Wulff, F. (2003). Humans, hydrology, and the distribution of inorganic nutrient loading to the ocean. Bioscience, 53(3), 235-245.

- Strickland, J. D. H., \& Parsons, T. R. (1972). A practical handbook of seawater analysis (2a. ed., pp. 71-80, 49-52, 263266). Ottawa, Canada: Fisheries Research Board.

- Swaney, D. P., Smith, S. V., \& Wulff, F. (2011). The LOICZ Biogeochemical Modeling Protocol and its application to estuarine ecosystems. En E. Wolanski, D. McLusky, D. Baird, \& A. Mehta (Eds.), Estuarine and Coastal Ecosystem Modeling (pp. 135-160). Amsterdam, Netherlands: Academic Press.

- Valenzuela-Siu, M., Arreola-Lizárraga, J. A., Sánchez-Carrillo S., \& Padilla- Arredondo, G. (2007). Flujos de nutrientes y metabolismo neto de la laguna costera Lobos, México. Hidrobiológica, 17(3), 193-202. 